\title{
On the Foundations of Eurhythmic Physics: A Brief Non Technical Survey
}

\author{
Paulo Castro $^{1}$, José Ramalho Croca ${ }^{1,2}$, Rui Moreira ${ }^{1}$, Mário Gatta ${ }^{1,3}$ \\ ${ }^{1}$ Center for Philosophy of Sciences of the University of Lisbon (CFCUL), Lisbon, Portugal \\ ${ }^{2}$ Department of Physics, University of Lisbon, Lisbon, Portugal \\ ${ }^{3}$ Centro de Investigação Naval (CINAV), Portuguese Naval Academy, Almada, Portugal
}

Email address:

jpcastro@fc.ul.pt (P. Castro), jncroca@fc.ul.pt (J. R. Croca), ranmoreira@gmail.com (R. Moreira), mariogatta@gmail.com (M. Gatta)

\section{To cite this article:}

Paulo Castro, José Ramalho Croca, Rui Moreira, Mário Gatta. On the Foundations of Eurhythmic Physics: A Brief Non Technical Survey. International Journal of Philosophy. Vol. 5, No. 6, 2017, pp. 50-53. doi: 10.11648/j.ijp.20170506.11

Received: October 9, 2017; Accepted: November 13, 2017; Published: February 3, 2018

\begin{abstract}
Eurhythmic Physics is a new approach to describe physical systems, where the concepts of rhythm, synchronization, inter-relational influence and non-linear emergence stand out as major concepts. The theory, still under development, is based on the Principle of Eurhythmy, the assertion that all systems follow, on average, the behaviors that extend their existence, preserving and reinforcing their structural stability. The Principle of Eurhythmy implies that all systems in Nature tend to harmonize or cooperate between themselves in order to persist, giving rise to more complex structures. This paper provides a brief non-technical introduction to the subject.
\end{abstract}

Keywords: Eurhythmic Physics, Principle of Eurhythmy, Non-Linearity, Emergence, Complex Systems, Cooperative Evolution

\section{Introduction}

Eurhythmic Physics [1] is a general inter-relational pilotwave theory, aiming to describe physical interactions at all scales in Nature. This attempt to understand Reality was first formulated in 2006 [2] and is still under development. It assumes that real physical systems are complex entities and that an emergent whole do not equate, in general, the mere aggregation of its parts. Given all reciprocal interactions, each constituent part will always produce structural changes on every other part, each part adapting, in a greater or lesser degree, to the remaining whole. It thus seems reasonable to acknowledge that the properties of an emergent system cannot, in general, be strictly inferred from the singular properties of its constituents parts, since these have lost their initial individuality.

In Eurhythmic Physics the concepts of mass and charge, assumed to be fundamental in classical and relativistic physics, no longer preserve this status, becoming relational concepts. Furthermore, Newtonian forces and space-time deformations are also not understood as fundamental, Newton's law of action-reaction being but a special case among a wider range of interactions. Acceptance of structural complexity leads to the idea that no real physical system may accurately be represented as an elementary material point, an object without extension and without an inner structure. This requirement is embedded in one of the most basic assumptions of Eurhythmic Physics: the one that states that each physical system is always composed of an highly localized and energetic structure called the acron (from the

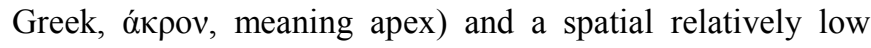
energetic extended field, called its theta wave. The acron is in permanent motion inside its self-generated theta wave field, being drawn to the regions of higher intensity in the field. The theta wave is thus acting as a piloting structure, influencing the acron's movement. This is the main reason why Eurhythmic Physics is considered a pilot-wave theory. The acron, on the other hand, must be understood as an oscillating device, permanently generating its theta wave, propagating throughout the medium. If, for instance, we are talking about light, the photon will be the acron and the usual Electromagnetic field a manifestation of the photon's theta wave. It should be mentioned that in most cases, photons behave as bosons, having a general tendency to aggregate. However, in other physical situations, photons can indeed 
behave like fermions [3], tending to draw apart. The attractiveness or repulsiveness an object exhibits towards others is therefore to be reasoned as depending on the conjugated effects that the surrounding physical systems have on an object, altering its theta wave. That is, each system set of properties depends upon the undulotory relational context, characterizing the medium where that system is immersed. When interacting theta waves, pertaining to two acrons, interfere as to create a region of overall lower intensity between the acra, the moving particles will draw away from each other. Otherwise, if the resulting field between the particles has an higher intensity, the former will attract. This explanatory scheme seems to be more general than the usual strategy, dismissing the need for forces, charge and mass and making possible the explanation of new phenomena or unexplained one. The so called «EM thrust», recently observed by a NASA research group, was explained using this same pilot-wave approach [4]. Another example concerns the so called Titius-Bode law, the fact that the average distances of the planets to the Sun follow an integer type law, suggesting the presence of an undulatory phenomena ordering the planets orbits. A pilot-wave gravity model was used to explain this regularity [5].

In Eurhythmic Physics interaction regimes between physical systems give rise to overall more stable structures, as a result of a coherence reinforcement of each system properties, due to the presence of all interacting entities. This overall process favors global cooperation from which all interacting parts gain structural stability, thus enduring in space and time. In fact, one of the theory basic assumptions, called the Principle of Eurhythmy, states that all physical systems have a propensity to adopt those overall behaviors that best favor each system stability. In this pilot-wave model the acron will tend to move to the regions of higher intensity in its theta wave field, because these will be the sites where the acron will best conserve its own energy. It is consistently supposed that the theta wave field is permanently maintained by its generating acron, a statement called the Visitation Hypothesis. An eurhythmic interaction between several systems is thought to be a cooperative process that reinforces or even creates new properties in each individual interacting system, pursuing the promotion of structural stability. Every complex acron is an oscillatory system that, no matter its scale, generates its own theta wave, producing a local ordering effect on what is called the subquantum medium. That is, the acron, be it an electron or a planet, induces a certain degree of wave coherence or organization on its immediate subquantum medium vicinity, becoming coupled to it. At the quantum scale, the acron in order to maintain or improve its overall stability, will favor stochastic paths along regions where its theta wave field has larger intensity, presenting random movements. At the macroscopic level, the systems will also prefer the regions of higher intensity of their theta waves. However, as a larger number of systems will be interacting, this will make the overall theta wave topology conform to the usual Newtonian and relativistic trajectories, as usually observed at such scales. As the number of interacting systems increases, so does the number of restrictions imposed upon all theta waves, making natural the assumption that, in general, an object will only move once affected by what is then understood to be an external cause, be it a force or a space-time deformed region. In fact, Eurhythmic Physics, sustains this to be the other way round. Newtonian and relativistic movements are but particular cases of a wider class of movements in Nature. An heuristic demonstration of what can possibly be the most general cinematic behavior of a system, perceived as an acron that is following and reinforcing its pilot wave, can be found in recent experiments involving bouncing droplets progressing on a vibrating veil of oil [6].

\section{Origins and Some Epistemological Consequences of Eurhythmic Physics}

Eurhythmic Physics was initially conceived as a nonlinear generalization of quantum physics [7], an intelligible alternative to the orthodox Copenhagen interpretation of Quantum Mechanics. This alternative view started from three main ideas. The first came from Louis de Broglie's double-solution theory, who, already in 1927, suggested at the celebrated Solvay conference, that quantum waves are real physical perturbations propagating the a subquantum medium. That is, not just abstract probabilistic waves of metaphysical origin that measures our state of knowledge about a physical system, as Niels Bohr championed. This last claim became known as the Copenhagen interpretation, still favored nowadays by standard Quantum Mechanics. The second idea, introduced by J. R. Croca, resulted from critical thinking about the statement that quantum waves are infinite entities, extending throughout unending space and unending time, as again championed by the Copenhagen School. As the waves are abstract, it would seem, at first sight, that such outstanding ubiquity would be logically acceptable. However, it so happens that the same abstract, metaphysical waves seem to be able to interact with slits and barriers, giving rise to interference patterns and somehow turning themselves into material objects, by means of the awareness powers of otherwise limited human observers. This somehow magical and mystical narrative is, in fact, an highly idealized interpretation coming from the so called Fourier ontology, relating to the mathematical techniques used in Fourier wave analysis, widely used in contemporary Quantum Mechanics formalism.

Contrarily to this view, Croca and the Lisbon School of Eurythmic Physics [9] would hold that quantum waves are real finite perturbations in the subquantum medium, decaying as one moves far apart from the generating acron. The mathematical formalism of Eurhythmic Physics consequently uses Morlet wavelets, in place of infinite harmonic plane waves. These infinite waves being a rather naive mathematical abstraction, once applied to physical reality. The wavelet formalism, once applied to physical description, seems to suggest the existence of different physical behaviors depending on the wavelet extension. In fact, situations from what can be called a quantum behavior, as for instants with 
the cases of entanglement, to physical behaviors where a wavelet extension of same order of magnitude as the dimensions of the acron, will allow simultaneously for local space-time coordination and causal description. Something Bohr would hold impossible in face of his celebrated Principle of Complementary. Indeed, this third fundamental idea behind Eurhythmic Physics comes from the fact that within the formalism of wavelet analysis one can derive a more general set of uncertainty relations trough wavelet packaging [10]. These general uncertainty relations formally contain Heisenberg relations as a particular case and allow for simultaneous refinement of the position and momentum values in a quantum measurement. The same being equally valid for the time-energy relation. The prediction for the measurement precision intervals of these physical quantities will now strongly depend on the size and duration of the theta wave involved in the experiment. This, by its turn, is related with the wavelet spatial frequency, making it possible to increase the experimental resolution beyond the limitations of common optical microscopes. One can see this happening in the case of scanning tunneling microscopes, where it is possible to obtain resolutions beyond the Heisenberg limits [11]. The two general uncertainty relations read:

$$
\begin{aligned}
\Delta p_{x} & =\frac{\hbar}{\Delta x} \sqrt{1-\Delta x^{2} / \sigma_{0 x}^{2}}, \\
\Delta E & =\frac{\hbar}{\Delta t} \sqrt{1-\Delta t^{2} / \sigma_{0 t}^{2}},
\end{aligned}
$$

The parameters $\sigma_{0 x}$ and $\sigma_{0 t}$ represen the wavelet spatial and temporal spreading. Once these values are comparatively larger than, respectively, the position uncertainty $\Delta x$ and the temporal uncertainty $\Delta t$, one obtains the usual Heisenberg relations, these being but a special limiting case of the former.

The authors have closely analyzed (1) and (2), reasoning about their epistemological consequences. Proper algebraic manipulation of the relations suggests the validity of what was named Completeness Principles in Physics [12], the first one stating that:

Nature is an ontological complete structure, without any logical mutual exclusiveness, that is, an ontological unified structure which is consistent and intelligible.

And, the second positing that:

Nature can be physically described at all scales using both a causal undulatory scheme and a space-time local scheme, with variable accuracy dependence between the two descriptions, such accuracy being given by the generalized uncertainty relations.

The two statements are, of course, consistent with the thesis that all physical systems in Nature comprise a corpuscular and an undolatory part that are both real. We have thus moved away from Bohr's original idealistic view to embrace a sort of unification possibility for physical theories. Even a planet can now be seen as a corpuscle surrounded by its own subquantum wave field, to be eventually equated with the classic gravitational field. It should be noted that as long as Niels Bohr Complementary Principle is accepted only on an epistemological level, and therefore not as an absolute statement about Nature, it will be a correct particular case of the second Completeness Principle.

As it may seem that things can never exhibit undulatory behaviors at the macroscopic scale, this in fact is not entirely true. Even if, in most cases, macroscopically observable undulatory behaviors do not express themselves under present experimental use, that does not mean that they aren't observable under other chosen conditions. This is, for instance, the case of the so-called Doubochinski's pendulum and similar coupled oscillatory systems, which show quantified amplitude behaviours [13]. Furthermore, once again, the authors have found that the Titius Bode regularity in the Solar System can be adequately explained using an undulatory formalism that involves stationary pilot-waves [5].

Eurhythmic Physics has also embedded in its formalism a nonlinear differential equation, similar to the usual Schrödinger equation but with an additional nonlinear term. The Schrödinger equation being a particular case of the former, whenever the non linear term is constant or null. The equation reading:

$$
-\frac{\hbar^{2}}{2 \mu} \nabla^{2} \theta+\frac{\hbar^{2}}{2 \mu} \frac{\nabla^{2}\left(\theta \theta^{*}\right)^{1 / 2}}{\left(\theta \theta^{*}\right)^{1 / 2}} \theta+V \theta=i \hbar \theta_{t}
$$

It has been shown that this Master equation, as it is so called, can be derived from both the Hamilton-Jacobi equation and the continuity equation. The Hamilton-Jacobi equation representing the local corpuscular aspect of the theory, namely, describing the properties of the acra, and the continuity equation addressing the extended wave like properties of the system [7]. The Master equation thus seems to result from a formal unification between the corpuscular and undulatory aspects of Nature, as de Broglie originally hypothesized. It should be mentioned that the nonlinear term $\frac{\hbar^{2}}{2 \mu} \frac{\nabla^{2}\left(\theta \theta^{*}\right)^{1 / 2}}{\left(\theta \theta^{*}\right)^{1 / 2}} \theta$ in (3), the so called quantum potential, may be interpreted as a mathematical statement for the existence of a reciprocal interaction between the corpuscle and its piloting wave. The gradient of the wave will be important for the corpuscle movement inside its theta wave, accordingly to the Principle of Eurhythmy. The quantum potential can also be seen as a statement for the existence of structural complexity in quantum systems, whose description, again, cannot be naively reduced to material points.

In face of the Hamilton-Jacobi equation, Eurhythmic Physics is usually introduced calling upon what is sometimes referred as weak teleological principles, where some quantity reaches an extreme, as in the Fermat Principle or the Maupertuis Principle. Indeed, these maximization principles can somehow be considered first historical theorizations of the Principle of Eurhythmy.

\section{The Fundamental Assumptions of Eurhythmic Physics}

There are five major assumptions in Eurhythmic Physics, also called Hyperphysics [2] since it shows promise for grand unification in the context of an undulatory framework. The assumptions read as follows: 
First assumption - Realism.

There is an objective Reality. This reality is observerindependent, yet, it is understood that the observer interacts with that very same reality, being able to change it and, of course, being changed by it in a greater or lesser degree.

Second assumption - Subquantum medium.

There is a basic physical natural chaotic medium named the subquantum medium. All physical processes occur in this natural chaotic medium.

Third assumption - Physical entities.

What are called physical entities that is, particles, fields and so on, are more or less stable local organizations of the basic chaotic subquantum medium.

Fourth assumption - Physical systems and the acron-theta wave structure.

What are called physical systems are stable organizations of the subquantum medium, composed of an extended region, the so called theta wave, where inside it there is a much smaller and localized structure of comparatively much higher energy named the acron.

Fifth assumption - The Principle of Eurhythmy.

This organizing principle states that the acron inside the theta field follows a stochastic path that in average leads to the regions where the intensity of the theta field is larger. It is understood that this behavior promotes the structural stability of the acron.

\section{Conclusion}

A non-technical introduction to Eurhythmic Physics has been briefly introduced. The authors main objective was to provide a new area for fruitful and though provoking ideas coming from Philosophers and other experts, and paving the way for further developments. The concepts of complexity, non-linear interaction, emergence of new properties and synchronization between systems, together with the Principle of Eurhythmy, show great promise once applied to other fields of knowledge. One may even think that cooperation between entities, promoting their stability, is the main trend by which evolution proceeds. This seems to reinforce the epistemological importance of symbiogenesis in natural evolution instead of the usual neo-Darwinistic mechanisms. From Physics to Biology and naturally to Human Sciences, one can try and look for possible relations between the proper rhythms of systems and their cooperation status in the physical, biological and social stages. A possibly fruitful trend of philosophical research would comprise the evaluation of what seems to be an ethical stance coming from Nature itself, as it promotes mutual reinforcing interactions, according to the Principle of Eurhythmy.

Under the unifying concept of Eurhythmy, the authors hope to contribute to the conceptual interchange between scientific disciplines, promoting interdisciplinary and the understanding of complex phenomena.

\section{Acknowledgements}

This work has been funded by FCT (Portugal) through CFCUL (project UID/FIL/00678/2013)

\section{References}

[1] J. R. Croca, The principle of eurhythmy a key to the unity of physics, Unity of Science, Nontraditional Approaches, Lisbon, October, 25-28, 2006. J. R. Croca, The principle of eurhythmy a key to the unity of physics, in Special Sciences and the Unity of Sciences, Eds. Pombo, O.; Torres, J. M.; Symons, J.; Rahman, S. (Eds.), Springer, 2012.

[2] J. R. Croca, Eurhythmic Physics, or Hyperphysics, The Unification of Physics, Lambert, Berlin, 2015.

[3] 1. Croca, J. R., Castro, P., Gatta, M., \& Gurriana, L. (2017). A Possible Explanation for the EM Drive Based on a Pilot Wave Theory. Journal of Applied Physical Science International, 8 (4), 145-151.

[4] Adenier, Guillaume; Bergli, Joakim; Thörn, Andreas Pierre \& Vistnes, Arnt Inge (2013). Observation of bosonic coalescence and fermionic anti-coalescence with indistinguishable photons. Proceedings of SPIE, the International Society for Optical Engineering. ISSN 0277-786X. 8832. doi: $10.1117 / 12.2024090$

[5] J. R. Croca, P. Castro, M. Gatta, A. Cardoso and R. Moreira, Pilot-wave gravity and the Titius-Bode Law, accepted. for publ. in New Horizons in Mathematical Physics, Isaac Scientific Publishing.

[6] Daniel M. Harris, Julien Moukhtar, Emmanuel Fort, Yves Couder, and John W. M. Bush, Wavelike statistics from pilotwave dynamics in a circular corral, Phys. Rev. E 88, 011001 (R) - Published 10 July 2013. See also dot/wave.org and Hydrodynamic quantum analogs.

[7] J. R. Croca, Towards a Nonlinear Quantum Physics, World Scientific, London, 2003.

[8] J. R. Croca, Quantum indeterminism: a direct consequence of Fourier ontology, Proc. SPIE 8832, The Nature of Light: What are Photons? V, 88320Y (October 1, 2013); doi:10.1117/12.2025291.

[9] Mazzola, Andrea. (2015) La controversia sulla teoria dei quanti e la scuola di Lisbona", in Physis, Casa Editrice Olschki of Florence on behalf of Domus Galiloeeana of Pisa, Vol. 50, n. 1-2.

[10] Croca, J. R. (2006). Beyond Heisenberg's Uncertainty Limits. In Gravitation and Cosmology: From the Hubble Radius to the Planck Scale: Proceedings of a Symposium in Honour of the 80th Birthday of Jean-Pierre Vigier. Springer Science \& Business Media.

[11] J. R. Croca, Counter Examples to the General Validity of Heisenberg Uncertainty Relations. Hadronic Journal, v. 22, p. 29-39, 1999, J. R. Croca, The Uncertainty Relations. Apeiron, v. 6, n. 3-4, p. 151-165, 1999 .

[12] P. Castro, J. R. Croca, M. Gatta, R. Moreira, Generalized Uncertainty Relations in Quantum Mechanics and the Principles of Completeness in Physics, accepted. for publ. in Physical Science International Journal, SCIENCEDOMAIN international.

[13] J. Tennenbaum, Amplitude Quantization as an Elementary Property of Macroscopic Vibrating Systems, $21^{\text {st }}$ Century Science and Technology, p. 50, Winter 2005-2006. 